\title{
Benzodiazepine drug use and cancer risk: a dose-response meta analysis of prospective cohort studies
}

\author{
Tao Zhang ${ }^{1, *}$, Xiaowen Yang ${ }^{2, *}$, Jianrui Zhou ${ }^{3}$, Pei Liu ${ }^{4}$, Hui Wang ${ }^{1}$, Anrong Li $^{1}$ and \\ Yi Zhou ${ }^{1}$ \\ ${ }^{1}$ Department of Neurosurgery, Taihe Hospital, Hubei University of Medicine, Shiyan, Hubei, 442000, China \\ ${ }^{2}$ Department of Clinical Laboratory, Taihe Hospital, Hubei University of Medicine, Shiyan, Hubei, 442000, China \\ ${ }^{3}$ Department of Rehabilitation Medicine, Taihe Hospital, Hubei University of Medicine, Shiyan, Hubei, 442000, China \\ ${ }^{4}$ Department of Dermatology, Taihe Hospital, Hubei University of Medicine, Shiyan, Hubei, 442000, China \\ *These authors contributed equally to this work and are co-first authors
}

Correspondence to: Tao Zhang, email: zhang_ta0@sina.com

Keywords: cancer, benzodiazepine, dose-response relationship, meta analysis

Received: July 19, $2017 \quad$ Accepted: September 21, $2017 \quad$ Published: October 19, 2017

Copyright: Zhang et al. This is an open-access article distributed under the terms of the Creative Commons Attribution License 3.0 (CC BY 3.0), which permits unrestricted use, distribution, and reproduction in any medium, provided the original author and source are credited.

\section{ABSTRACT}

Conflicting results identifying the relationship between benzodiazepine drug use and cancer risk. Therefore, we conducted a dose-response meta-analysis of prospective cohort studies to clarify and quantitative assessed the relationship between benzodiazepine drug use and cancer risk. Up to July 2017, 22 original publications were included in current meta-analysis. Our results showed statistically significant association between benzodiazepine drug use and cancer risk (RR:1.25; 95\% CI, 1.15-1.36). Subgroup analysis showed benzodiazepine using was associated with significantly a higher risk of breast cancer (RR:1.15; 95\% CI, 1.05-1.26), ovarian cancer (RR:1.17; 95\% CI, 1.09-1.25), colon cancer (RR:1.07; 95\% CI, 1.02-1.13), renal cancer (RR:1.31; 95\% CI, 1.15-1.49), malignant melanoma (RR:1.10; 95\% CI, 1.03-1.17), brain cancer (RR:2.06; 95\% CI, 1.76-2.43), esophagus cancer (RR:1.55; 95\% CI, 1.30-1.85), prostate cancer (RR:1.26; 95\% CI, 1.16-1.37), liver cancer (RR:1.22; 95\% CI, 1.13-1.31), stomach cancer (RR:1.17; 95\% CI, 1.03-1.32), pancreatic cancer (RR:1.39; 95\% CI, 1.17-1.64) and lung cancer (RR:1.20; 95\% CI, 1.12-1.28). Furthermore, a significant dose-response relationship was observed between benzodiazepine drug use and cancer risk (likelihood ratio test, $P<0.001$ ). Our results showed per $500 \mathrm{mg} /$ year, per 5 year of time since first using, per 3 prescriptions and per 3 year of duration incremental increase in benzodiazepine drug use was associated with a $17 \%, 4 \%, 16 \%$ and $5 \%$ in cancer risk increment. Considering these promising results, increasing benzodiazepine using might be harmful for health.

\section{INTRODUCTION}

Cancer is the second most frequently leading cause that caused over 8.8 million deaths worldwide in 2015 [1], and the World Health Organization predicts 14.1 million people are expected to develop cancer annually [2]. Cancer represents a heavy social health problem and economic burden, and costs on patients. The etiology of cancer involves both genetic and environmental factors. Multiple lines of evidence have demonstrated that benzodiazepine drug use is the risk factors for cancer regardless of laboratory studies or animal studies [3, 4].

Benzodiazepines are the derivatives of 1 , 4-benzodiazepines, including diazepam, flurazepam, chlorazepam, oxazepam and triazolam. The main function of benzodiazepine is inducing feelings of calm, drowsiness and sleep, and have been widely used in clinical, mainly to treat anxiety and insomnia. Benzodiazepines has been one of the most commonly used drugs in the general population for nearly 50 years, with the use of older people 
worldwide ranging from 10 to 42 percent. In the United States, about 6 to 10 percent of adults use benzodiazepines in 2010, while in Europe, some regions have found a higher proportion $[5,6]$. Therefore, it is important to evaluate the benzodiazepines drugs safety.

Previous studies have examined the correlation between benzodiazepines drug use and cancer risk [7-28]. However, the result remains controversial. Additionally, no study to quantitative assessed benzodiazepines drug use in relation to cancer risk. Thus, we performed this doseresponse meta-analysis to clarify and quantitative assessed the correlation between benzodiazepines drug use and cancer risk.

\section{RESULTS}

\section{Literature search results}

Figure 1 shows literature research and selection. A total of 2304 studies from PubMed, 2638 studies from Embase and 2516 studies from Web of Science. After exclusion of duplicates and studies that did not fulfill the inclusion criteria, 22 studies were chosen, and the data were extracted. These studies were published update to July 2017.

\section{Study characteristics}

The characteristics of the included studies of benzodiazepines drug use and cancer risk are shown in the Tables 1 and 2. Results in different subgroups were treated as two separate reports. Finally, twenty-seven independent reports from twenty two studies investigated the association between benzodiazepines drug use and cancer risk. Among the selected reports, twenty-two are from Caucasia and five from Asia. A total of 2482625 participants with 312203 incident cases from seven countries were included in this meta-analysis.

\section{Benzodiazepines drug use and overall cancer risk}

Twenty-seven independent reports from twenty two studies investigated the association between between benzodiazepines drug use and cancer risk. Compared with no benzodiazepines drug use, benzodiazepines drug use is significantly associated with a higher risk of cancer risk (RR:1.25; 95\% CI, 1.15-1.36; $P<0.001$ ) (Table 3). Furthermore, benzodiazepines drug use is significantly associated with cancer risk in Caucasia (RR:1.21; 95\% CI, 1.05-1.39; $P<0.001$ ) (Table 3) and Asia (RR:1.36; 95\% CI, 1.16-1.59; $P<0.001$ ) (Table 3). Additionally, benzodiazepines drug use is significantly associated with cancer risk in female (RR:1.14; 95\% CI, 1.04-1.24; $P=$ 0.004 ) (Table 3) but not in male (RR:1.12; 95\% CI, 0.96 $1.30 ; P=0.154$ ) (Table 3). That may be because there isn't enough data in male.

\section{Benzodiazepine drug use and the risk of cancer by type of cancer}

Eleven independent reports from seven studies investigated the association between benzodiazepines drug use and breast cancer. Compared with no benzodiazepines drug use, benzodiazepines drug use is significantly associated with a higher risk of breast cancer (RR:1.15; 95\% CI, 1.05-1.26; $P<0.001$ ) (Table 4). Furthermore, benzodiazepines drug use is significantly associated with breast cancer risk in Caucasia (RR:1.17; 95\% CI, $1.08-1.26 ; P<0.001$ ) (Table 4) and Asia (RR:1.09; 95\% CI, 1.03-1.16; $P<0.001$ ) (Table 4). Eight independent reports from six studies investigated the association between benzodiazepines drug use and ovarian cancer risk. Compared with no benzodiazepines drug use, benzodiazepines drug use is significantly associated with a higher risk of ovarian cancer (RR:1.17; 95\% CI, 1.09$1.25 ; P<0.001)$ (Table 4). Furthermore, benzodiazepines drug use is significantly associated with ovarian cancer risk in Caucasia (RR:1.22; 95\% CI, 1.15-1.30; $P<$ 0.001) (Table 4) and Asia (RR:1.11; 95\% CI, 1.05-1.17; $P<0.001$ ) (Table 4). Additionally, Compared with no benzodiazepines drug use, benzodiazepines drug use is significantly associated with a higher risk of colon cancer (RR:1.07; 95\% CI, 1.02-1.13; $P<0.001)$ (Table4), renal cancer (RR:1.31; 95\% CI, 1.15-1.49; $P<0.001)$ (Table4), malignant melanoma (RR:1.10; 95\% CI, 1.03-1.17; $P<$ 0.001) (Table 4), brain cancer (RR:2.06; 95\% CI, 1.762.43; $P<0.001)$ (Table 4), esophagus cancer (RR:1.55; 95\% CI, 1.30-1.85; $P<0.001$ ) (Table 4), prostate cancer (RR:1.26; 95\% CI, 1.16-1.37; $P<0.001$ ) (Table 4), liver cancer (RR:1.22; 95\% CI, 1.13-1.31; $P<0.001$ ) (Table 4), stomach cancer (RR:1.17; 95\% CI, 1.03-1.32; $P<0.001$ ) (Table 4), pancreatic cancer (RR:1.39; 95\% CI, 1.17-1.64; $P<0.001$ ) (Table 4), lung cancer (RR:1.20; 95\% CI, 1.121.28; $P<0.001$ ) (Table 4).

\section{Subgroup analyses}

Subgroup analysis was performed to check the stability of the primary outcome. Subgroup meta-analyses in study design, study quality, number of participants and number of cases showed consistent findings (Tables 3, 4).

\section{Dose-response between benzodiazepines drug use and cancer risk}

Using restricted cubic spline function, the test for a nonlinear dose-response relationship was significant (likelihood ratio test, $P<0.001$ ), suggesting curvature in the relationship, increasing per $500 \mathrm{mg} /$ year of benzodiazepine drug use was associated with a $17 \%$ increment of cancer risk, the summary relative risk of cancer risk for an per $500 \mathrm{mg} /$ year of benzodiazepine drug use was $1.17(95 \% \mathrm{CI}: 1.02-1.35, P=0.022)$ 
(Figure 2). In addition, increasing benzodiazepine drug use (per 3 prescriptions increment) was associated with a $16 \%$ increment in cancer risk, the summary relative risk of cancer risk for an per 3 prescriptions of benzodiazepine drug use was 1.16 (95\% CI: 1.11-1.22, $P<0.001$ ) (Figure 2). Furthermore, increasing per 3 year of duration of benzodiazepine drug use was associated with a $5 \%$ increment of cancer risk, the summary relative risk of cancer risk for an per 3 year of duration of benzodiazepine drug use was 1.05 (95\% CI: 1.02-1.09, $P=0.042$ ) (Figure 2). Additionally, increasing per 5 year of time since first benzodiazepine drug use was associated with a $4 \%$ increment of cancer risk, the summary relative risk of cancer risk for an per 5 year of time since first benzodiazepine drug use was 1.04 (95\% CI: 1.01-1.07, $P=0.003$ ) (Figure 2).

\section{Sensitivity analysis}

Sensitivity analysis was conducted to assess the stability of the results. The results show the results were stable in Supplementary Figure 1.

\section{Publication bias}

Each studies in this meta-analysis were performed to evaluate the publication bias by both Begg's funnel plot and Egger's test. $P>0.05$ was considered no publication bias. The results show no obvious evidence of publication bias was found in the associations between benzodiazepine drug use and cancer risk (Supplementary Table 1).

\section{DISCUSSION}

Benzodiazepines are widely used to treat seizures, anxiety, insomnia, and panic disorder. In recent years there has been constant evidence that benzodiazepines are associated with cancer risk in vitro laboratory and animal studies. Several animal studies reported that benzodiazepines increase the risk of thyroid cancer [29] or liver cancer [3] through inhibiting apoptosis and stimulating tumor cell proliferation. In the meantime, several observational epidemiological studies reported that there was no link between benzodiazepines drug use and the risk cancer. However, recent cohort studies revealed that the use of benzodiazepines drugs was associated with

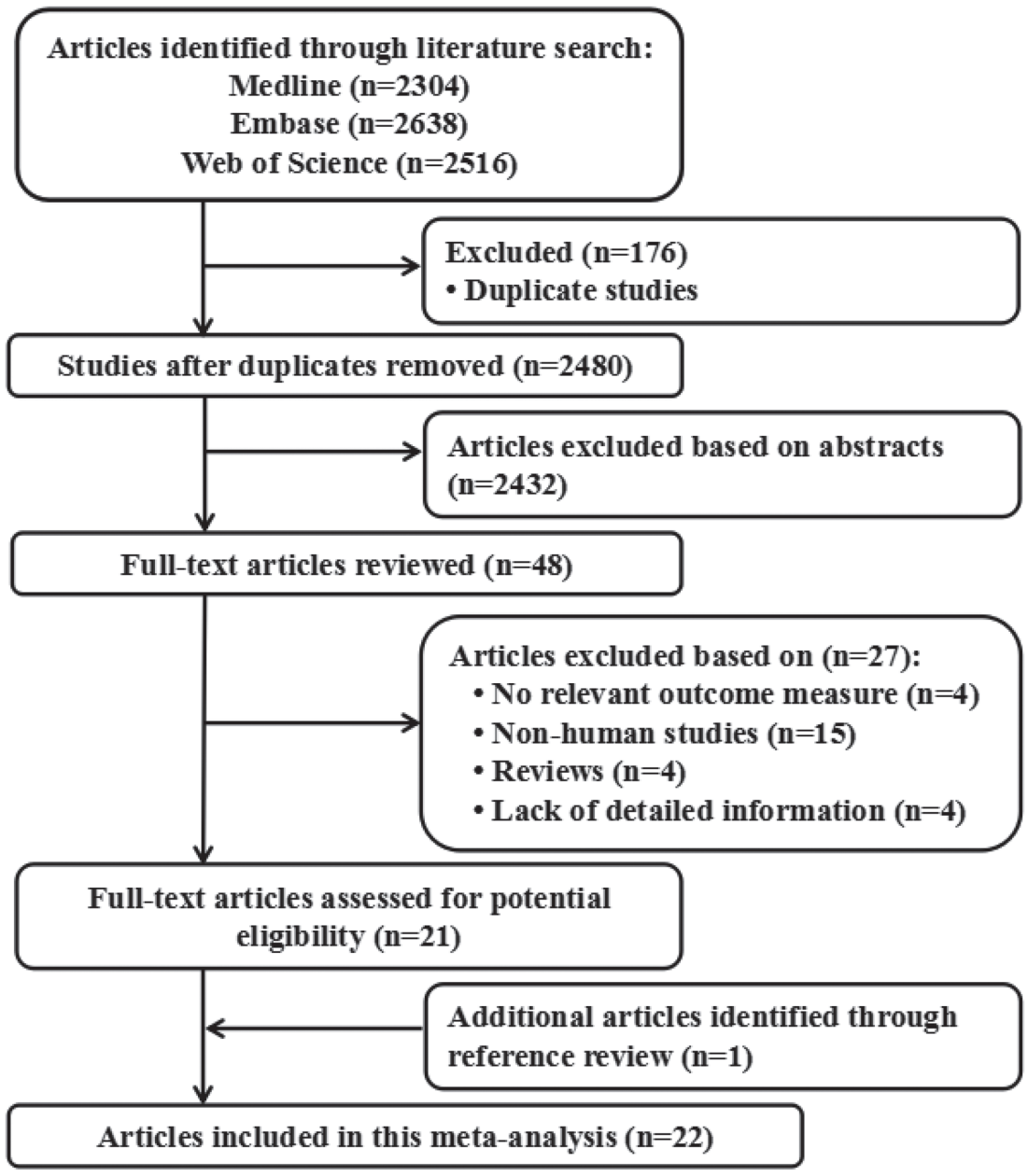

Figure 1: Flow diagram of the study selection process. 
Table 1: Characteristics of participants in included studies of benzodiazepine using in relation to risk of cancer

\begin{tabular}{|c|c|c|c|c|c|c|c|}
\hline Author (year) & Study design & Country & $\begin{array}{c}\text { Sex of } \\
\text { population }\end{array}$ & $\begin{array}{l}\text { Age at } \\
\text { baseline } \\
\text { (years) }\end{array}$ & $\begin{array}{c}\text { No of } \\
\text { participants }\end{array}$ & Endpoints (cases) & $\begin{array}{c}\text { Quality } \\
\text { score }\end{array}$ \\
\hline Coogan et al. (2000) & Case-control & United States & Mix & $<69$ & 3740 & Ovarian cancer (748) & 6 \\
\hline Dublin et al. (2002) & Case-control & United States & Mix & $35-79$ & 1104 & Ovarian cancer (314) & 7 \\
\hline Fortuny et al. (2007) & Case-control & United States & Mix & 66.8 & 4110 & $\begin{array}{c}\text { Esophageal adenocarcinoma (163) } \\
\text { Gastric cardia adenocarcinoma (176) } \\
\text { Esophageal squamous cell carcinoma (114) } \\
\text { Gastric non-cardia adenocarcinoma (320) }\end{array}$ & 8 \\
\hline Friedman et al. (1998) & Case-control & United States & Mix & $30-79$ & 4403 & Colon cancer (1960) & 7 \\
\hline Halapy et al. (2006) & Case-control & Canada & Female & $25-74$ & 6195 & Breast cancer (3133) & 7 \\
\hline Hardell et al. (1996) & Case-control & Sweden & Mix & NA & 987 & Colon cancer (329) & 5 \\
\hline Harlow et al. (1995) & Case-control & United States & Mix & $18-80$ & 904 & Ovarian cancer (450) & 7 \\
\hline Harnod et al. (2014) & $\begin{array}{l}\text { Prospective } \\
\text { cohort }\end{array}$ & China & Mix & $\geq 20$ & 62050 & Brain cancer (274) & 7 \\
\hline Jaussent et al. (2013) & $\begin{array}{l}\text { Prospective } \\
\text { cohort }\end{array}$ & France & Mix & $65-95$ & 6696 & All cancers (1454) & 8 \\
\hline Kao et al. (2012) & $\begin{array}{l}\text { Prospective } \\
\text { cohort }\end{array}$ & China & Mix & 47.9 & 119239 & All cancers $(3520)$ & 6 \\
\hline Kaufman et al. (1982) & Case-control & $\begin{array}{l}\text { Canada,United } \\
\text { States and Israel }\end{array}$ & Female & $<70$ & 1964 & Breast cancer $(1236)$ & 6 \\
\hline Kleinerman et al. (1984) & Case-control & United States & Female & $\geq 35$ & 2221 & Breast cancer $(1075)$ & 7 \\
\hline Kaufman et al. (1990) & Case-control & $\begin{array}{c}\text { United States } \\
\text { Canada }\end{array}$ & Female & $18-69$ & 5009 & Breast cancer $(3078)$ & 7 \\
\hline Rosenberg et al (1995) & Case-control & United States & Mix & $18-69$ & 6077 & All cancers $(3820)$ & 7 \\
\hline Westerdahl et al. (1996) & Case-control & Sweden & Mix & $15-75$ & 1040 & Malignant melanoma (400) & 7 \\
\hline Lagergren et al. (2000) & Case-control & Sweden & Mix & $<80$ & 1009 & Esophageal cancer (189) & 7 \\
\hline Pogoda et al. (2005) & Case-control & United States & Mix & $25-75$ & 824 & Acute myeloid leukemia (412) & 7 \\
\hline Lagergren et al. (2006) & Case-control & United States & Female & $21-84$ & 870 & Malignant melanoma (179) & 6 \\
\hline Kripke et al. (2012) & $\begin{array}{l}\text { Prospective } \\
\text { cohort }\end{array}$ & United States & Female & $\geq 18$ & 25750 & All cancers (2076) & 8 \\
\hline Pottegardet al. (2012) & Case-control & Denmark & Mix & $56-74$ & 1214099 & All cancers $(149360)$ & 8 \\
\hline Iqbal et al. (2015) & Case-control & China & Mix & $\geq 20$ & 255000 & All cancers (42500) & 8 \\
\hline Thygesen et al. (2017) & Case-control & Denmark & Mix & $18-85$ & 759334 & All cancers (94923) & 7 \\
\hline
\end{tabular}

an increased risk of cancer, and the benzodiazepine users were exposed to the risk of benign brain tumor about three times higher than the non-benzodiazepine users. Collectively, these data suggest that benzodiazepines drug use may play an important role in cancer risk, but presented controversial results. Thus, we performed this meta-analysis, aiming to study the role of benzodiazepines in cancer risk and to explain the possible reasons for controversial results.

In the current meta-analysis was based on 22 casecontrol or cohort study, with 2482625 participants with 312203 incident cases from seven countries. Thus, this meta analysis provides the most up-to-date epidemiological evidence supporting benzodiazepines drug use is harmful for health. A dose-response analysis revealed that increasing per $500 \mathrm{mg} / \mathrm{year}$ of benzodiazepine drug use was associated with a $17 \%$ increment of cancer risk. In addition, increasing benzodiazepine drug use (per 3 prescriptions increment) was associated with a $16 \%$ increment in cancer risk. Furthermore, increasing per 3 year of duration of benzodiazepine drug use was associated with a $5 \%$ increment of cancer risk. Additionally, increasing per 5 year of time since first benzodiazepine drug use was associated with a $4 \%$ increment of cancer risk. Subgroup meta-analyses by various factors also showed consistent findings.

Several plausible pathways may reasonable for the relationship between benzodiazepine drug use and cancer risk. The influence of chronic inflammation on cancer development is one possible pathway. Benzodiazepine drug use might increase the levels of inflammation mediators [30], which can increase the risk of cancer 
Table 2: Outcomes and covariates of included studies of benzodiazepine using in relation to risk of cancer

\begin{tabular}{|c|c|c|c|c|}
\hline Author (year) & Endpoints & Data source & Category and relative risk $(95 \% \mathrm{CI})$ & Covariates in fully adjusted model \\
\hline Coogan et al. (2000) & Ovarian cancer (748) & Population-based & $\begin{array}{c}\text { Duration of regular use } \\
0 \text { using, } 1.0 \text { (reference); } 0 \text { to } 5 \text { years using, } 0.9(0.6 \text {, } \\
1.3) \text {; more than } 5 \text { years using, } 1.1(0.5,2.3)\end{array}$ & $\begin{array}{l}\text { Adjusted for age, study center, and interview } \\
\text { year. }\end{array}$ \\
\hline Dublin et al. (2002) & Ovarian cancer (314) & Self-administered & $\begin{array}{c}\text { Duration of regular use } \\
0 \text { using, } 1.0 \text { (reference); } 4 \text { months using, } 0.76(0.52, \\
1.10) ; 6 \text { months using, } 0.70(0.47,1.0) \\
\text { Number of prescriptions } \\
0 \text { prescriptions } 1.0 \text { (reference); } 1,0.65(0.42,1.0) ; 2 \\
\text { to } 4,0.91(0.57,1.5) ;>5,0.68(0.42,1.1)\end{array}$ & Adjusted for age and reference date. \\
\hline Fortuny et al. (2007) & $\begin{array}{l}\text { Esophageal } \\
\text { adenocarcinoma (163) } \\
\text { Gastric cardia } \\
\text { adenocarcinoma (176) } \\
\text { Esophageal squamous } \\
\text { cell carcinoma (114) } \\
\text { Gastric non-cardia } \\
\text { adenocarcinoma (320) }\end{array}$ & Population-based & $\begin{array}{c}\text { Esophageal adenocarcinoma } \\
\text { Number of prescriptions } \\
0 \text { prescriptions } 1.0 \text { (reference) }<<1,1.0(0.5,1.7) ; 1 \text { to } \\
5,0.8(0.5,1.5) ;>5,0.8(0.4,1.5) \\
\text { Gastric cardia adenocarcinoma } \\
5 \text { prescriptions } 1.0 \text { (reference); }<1,0.8(0.5,1.3) ; 1 \text { to } \\
5,0.4(0.1,0.9) ;>5,0.6(0.3,1.1) \\
\text { Esophageal squamous cell carcinoma } \\
0 \text { prescriptions } 1.0 \text { (reference); }<1,0.6(0.3,1.2) ; 1 \text { to } \\
5,0.8(0.4,1.9) ;>5,1.7(0.9,3.1) \\
\text { Gastric non-cardia adenocarcinoma } \\
0 \text { prescriptions } 1.0 \text { (reference); }<1,0.6(0.4,0.8) ; 1 \text { to } \\
5,0.6(0.4,1.0) ;>5,0.5(0.3,0.8)\end{array}$ & $\begin{array}{c}\text { Adjusted for age, sex, HMO, years of } \\
\text { enrollment in the HMO, race at HFHS, and } \\
\text { adjusted for use of drug classes other than the } \\
\text { studied one. }\end{array}$ \\
\hline Friedman et al. (1998) & Colon cancer (1960) & Population-based & $\begin{array}{c}\text { Duration of regular use } \\
0 \text { using, } 1.0 \text { (reference); less than 1years using, } 0.9 \\
(0.7,1.3) ; 1 \text { to } 5 \text { years using, } 1.2(0.8,1.8) \text {; more than } \\
5 \text { years using, } 0.7(0.3,1.2)\end{array}$ & $\begin{array}{c}\text { Age, sex, aspirin and NSAID use,family } \\
\text { history of colorectal cancer,body mass } \\
\text { index,total calorie, fiber and calcium intake, } \\
\text { physical activity, cigarette smoking and alcohol } \\
\text { use }\end{array}$ \\
\hline Halapy et al. (2006) & Breast cancer (3133) & Self-administered & $\begin{array}{c}\text { Duration of regular use } \\
0 \text { using, 1.0 (reference); less than 1years using, } 0.90 \\
(0.66,1.22) ; 1 \text { to } 6 \text { years using, } 1.23(0.90,1.69) \\
\text { more than } 6 \text { years using, } 1.32(0.95,1.84)\end{array}$ & $\begin{array}{l}\text { Adjusted for age, family history of breast } \\
\text { cancer, and benign breast cysts. }\end{array}$ \\
\hline Jaussent et al. (2013) & Colon cancer (329) & Population-based & $\begin{array}{c}\text { Number of prescriptions } \\
0 \text { prescriptions } 1.0 \text { (reference); } 1,0.96(0.74,1.25) ;> \\
2,0.93(0.50,1.71)\end{array}$ & $\begin{array}{l}\text { Adjusted for age, study center, and gender; } \\
\text { badjusted for all covariates in model } 1 \text { plus } \\
\text { high level of education, confinement, alcohol } \\
\text { intake, smoking status, history of cardio- } \\
\text { cerebrovascular disease, respiratory disease, } \\
\text { Mini Mental State Examination score, body } \\
\text { mass index, hypertension and diabetes mellitus, } \\
\text { depressive symptoms, antidepressants use. }\end{array}$ \\
\hline Kaufman et al. (1982) & Ovarian cancer $(450)$ & Self-administered & $\begin{array}{c}\text { Duration of regular use } \\
0 \text { using, } 1.0 \text { (reference); less than } 5 \text { years using, } \\
0.9(0.4,1.7) \text {; more than } 5 \text { years using, } 1.0(0.4,2.6)\end{array}$ & $\begin{array}{l}\text { Age, geographical region, years of education, } \\
\text { religion, } \\
\text { parity, age at first pregnancy, menopausal } \\
\text { status, age at menopause, history of breast } \\
\text { cancer in the mother or sisters, } \\
\text { alcohol consumption, number of visits to a } \\
\text { doctor in the preceding year, total number of } \\
\text { hospital admissions, and year of interview }\end{array}$ \\
\hline Kaufman et al. (1990) & Brain cancer (274) & Population-based & $\begin{array}{c}\text { US } \\
\text { Duration of regular use } \\
0 \text { using, 1.0 (reference); less than } 5 \text { years using, } 1.2 \text { ( } \\
0.7,2.3) \text {; more than } 5 \text { years using, } 0.7(0.3,1.6) \\
\text { Canada } \\
\text { Duration of regular use } \\
0 \text { using, } 1.0 \text { (reference); less than } 5 \text { years using, } 0.7 \\
(0.4,1.3) \text {; more than } 5 \text { years using, } 1.0(0.5,2)\end{array}$ & $\begin{array}{c}\text { Age, Alcohol consumption, medical } \\
\text { history,lifetime history of medication use, use } \\
\text { of muscle relaxants, tranqulizers, psychiatric } \\
\text { drugs, insomnia and pain }\end{array}$ \\
\hline Lagergren et al. (2000) & All cancers $(1454)$ & Population-based & $\begin{array}{c}\text { Duration of regular use } \\
0 \text { using, } 1.0 \text { (reference); less than } 5 \text { years using, } 0.8 \\
(0.6,3.2) \text {; more than } 5 \text { years using, } 1.5(0.7,2.9)\end{array}$ & $\begin{array}{c}\text { Age, sex, body mass index ,tobacco } \\
\text { smoking, alcohol use, socioeconomic } \\
\text { status (years of formal education), and intake } \\
\text { of fruit and vegetables }\end{array}$ \\
\hline Pottegard et al. (2012) & All cancers $(3520)$ & Self-administered & $\begin{array}{c}\text { Duration of regular use } \\
0 \text { using, } 1.0 \text { (reference); less than lyears using, } 1.03 \\
(1.02,1.05) ; 1 \text { to } 3 \text { years using, } 1.05(1.02,1.08) ; 3 \text { to } \\
7 \text { years using, } 1.07(1.03,1.12) ; \text { more than } 7 \text { years } \\
\text { using, } 1.11(1.01,1.23)\end{array}$ & $\begin{array}{c}\text { Age, gender, use of aspirin, non- } \\
\text { aspirinNSAIDs, 5-areductase inhibitors, } \\
\text { statins, angiotensin-II } \\
\text { antagonists, oral } \\
\text { contraceptives and hormone supplements, } \\
\text { antidepressants, } \\
\text { antipsychotics, diagnoses of inflammatory } \\
\text { bowel disease, COPD, diabetes, } \\
\text { alcohol abuse and Charlson Comorbidity Index } \\
\text { score }\end{array}$ \\
\hline
\end{tabular}


Table 3: Stratified analyses of relative risk of cancer

\begin{tabular}{|c|c|c|c|c|c|}
\hline & No of reports & Relative risk (95\% CI) & P for heterogeneity & $I^{2}$ & $P$ for test \\
\hline Total & 27 & $1.25(1.15-1.36)$ & 0.000 & $78.8 \%$ & $<0.001$ \\
\hline \multicolumn{6}{|c|}{ Subgroup analyses for cancer } \\
\hline \multicolumn{6}{|c|}{ Study location } \\
\hline Caucasia & 22 & $1.21(1.05-1.39)$ & 0.000 & $66.3 \%$ & 0.008 \\
\hline Asia & 5 & $1.36(1.16-1.59)$ & 0.000 & $91.0 \%$ & $<0.001$ \\
\hline \multicolumn{6}{|l|}{ Gender } \\
\hline Female & 10 & $1.14(1.04-1.24)$ & 0.015 & $56.2 \%$ & 0.004 \\
\hline Male & 2 & $1.12(0.96-1.30)$ & 0.591 & $0.0 \%$ & 0.154 \\
\hline \multicolumn{6}{|l|}{ Study design } \\
\hline Case-control & 21 & $1.15(1.05-1.26)$ & 0.000 & $61.5 \%$ & 0.002 \\
\hline Cohort & 6 & $1.43(1.12-1.83)$ & 0.000 & $92.0 \%$ & $<0.001$ \\
\hline \multicolumn{6}{|l|}{ Study quality } \\
\hline Score $\geq 7$ & 20 & $1.27(1.13-1.42)$ & 0.000 & $84.0 \%$ & $<0.001$ \\
\hline Score $<7$ & 7 & $1.20(1.12-1.29)$ & 0.484 & $0.0 \%$ & $<0.001$ \\
\hline \multicolumn{6}{|l|}{ No of participants } \\
\hline$\geq 10000$ & 7 & $1.43(1.12-1.83)$ & 0.000 & $92.0 \%$ & $<0.001$ \\
\hline$<10000$ & 20 & $1.15(1.05-1.26)$ & 0.000 & $61.5 \%$ & 0.002 \\
\hline \multicolumn{6}{|l|}{ No of cases } \\
\hline$\geq 1000$ & 13 & $1.26(1.16-1.37)$ & 0.000 & $79.5 \%$ & $<0.001$ \\
\hline$<1000$ & 14 & $1.22(1.13-1.31)$ & 0.000 & $62.9 \%$ & $<0.001$ \\
\hline \multicolumn{6}{|l|}{ Types of benzodiazepine } \\
\hline Long-acting (Diazepam) & 8 & $1.08(0.94-1.24)$ & 0.075 & $50.1 \%$ & 0.306 \\
\hline Intermediate-acting & 6 & $1.21(1.16-1.23)$ & 0.000 & $75.3 \%$ & $<0.001$ \\
\hline Short-acting & 3 & $1.16(1.07-1.26)$ & 0.671 & $0.0 \%$ & $<0.001$ \\
\hline \multicolumn{6}{|c|}{ Duration of benzodiazepine use } \\
\hline 0 years & 8 & 1 & & & \\
\hline$<5$ years & 8 & $1.09(1.05-1.14)$ & 0.000 & $64.6 \%$ & $<0.001$ \\
\hline$\geq 5$ years & 8 & $1.20(1.16-1.23)$ & 0.000 & $72.8 \%$ & $<0.001$ \\
\hline \multicolumn{6}{|l|}{ Cumulative yearly dose } \\
\hline Lower & 3 & 1 & & & \\
\hline Moderate & 3 & $1.59(1.26-2.00)$ & 0.000 & $62.8 \%$ & $<0.001$ \\
\hline Highest & 3 & $2.93(2.45-3.52)$ & 0.000 & $96.6 \%$ & $<0.001$ \\
\hline \multicolumn{6}{|l|}{ Number of prescriptions } \\
\hline Lower & 6 & 1 & & & \\
\hline Highest & 6 & $1.12(1.03-1.22)$ & 0.069 & $51.2 \%$ & $<0.001$ \\
\hline \multicolumn{6}{|l|}{ Time since first use } \\
\hline 0 years & 5 & 1 & & & \\
\hline$<10$ years & 5 & $1.14(1.05-1.24)$ & 0.753 & $0.0 \%$ & $<0.001$ \\
\hline$\geq 10$ years & 5 & $1.23(1.13-1.33)$ & 0.175 & $36.9 \%$ & $<0.001$ \\
\hline \multicolumn{6}{|c|}{ Time since last benzodiazpine use } \\
\hline 0 years & 2 & 1 & & & \\
\hline$<1$ years & 2 & $0.97(0.79-1.20)$ & 0.381 & $0.0 \%$ & 0.781 \\
\hline$\geq 1$ years & 2 & $1.16(0.87-1.56)$ & 0.889 & $0.0 \%$ & 0.322 \\
\hline
\end{tabular}


Table 4: Stratified analyses of relative risk of different cancer

\begin{tabular}{|c|c|c|c|c|c|}
\hline & No of reports & Relative risk (95\% CI) & P for heterogeneity & $\mathbf{I}^{2}$ & $P$ for test \\
\hline Breast cancer & 11 & $1.15(1.05-1.25)$ & 0.345 & $17.3 \%$ & $<0.001$ \\
\hline \multicolumn{6}{|c|}{ Subgroup analyses for Breast cancer } \\
\hline \multicolumn{6}{|l|}{ Study location } \\
\hline Caucasia & 7 & $1.17(1.08-1.26)$ & 0.258 & $25.6 \%$ & $<0.001$ \\
\hline Asia & 4 & $1.09(1.03-1.16)$ & 0.631 & $0.0 \%$ & $<0.001$ \\
\hline \multicolumn{6}{|l|}{ Study design } \\
\hline Case-control & 8 & $1.05(1.01-1.09)$ & 0.214 & $23.6 \%$ & $<0.001$ \\
\hline Cohort & 3 & $1.19(1.12-1.28)$ & 0.447 & $0.0 \%$ & $<0.001$ \\
\hline \multicolumn{6}{|l|}{ Study quality } \\
\hline Score $\geq 7$ & 7 & $1.06(1.02-1.10)$ & 0.215 & $24.3 \%$ & $<0.001$ \\
\hline Score $<7$ & 4 & $1.19(1.11-1.28)$ & 0.474 & $0.0 \%$ & $<0.001$ \\
\hline \multicolumn{6}{|l|}{ No of participants } \\
\hline$\geq 10000$ & 8 & $1.05(1.01-1.09)$ & 0.214 & $23.6 \%$ & $<0.001$ \\
\hline$<10000$ & 3 & $1.19(1.12-1.28)$ & 0.447 & $0.0 \%$ & $<0.001$ \\
\hline \multicolumn{6}{|l|}{ No of cases } \\
\hline$\geq 1500$ & 9 & $1.07(1.02-1.12)$ & 0.101 & $33.6 \%$ & $<0.001$ \\
\hline$<1500$ & 2 & $1.18(1.10-1.26)$ & 0.673 & $0.0 \%$ & $<0.001$ \\
\hline Ovarian cancer & 8 & $1.17(1.09-1.25)$ & 0.000 & $74.5 \%$ & $<0.001$ \\
\hline \multicolumn{6}{|c|}{ Subgroup analyses for Ovarian cancer } \\
\hline \multicolumn{6}{|l|}{ Study location } \\
\hline Caucasia & 4 & $1.22(1.15-1.30)$ & 0.008 & $80.2 \%$ & $<0.001$ \\
\hline Asia & 4 & $1.11(1.05-1.17)$ & 0.657 & $0.0 \%$ & $<0.001$ \\
\hline \multicolumn{6}{|l|}{ Study design } \\
\hline Case-control & 5 & $1.14(1.02-1.28)$ & 0.000 & $84.4 \%$ & 0.012 \\
\hline Cohort & 3 & $1.19(1.12-1.28)$ & 0.447 & $0.0 \%$ & $<0.001$ \\
\hline \multicolumn{6}{|l|}{ Study quality } \\
\hline Score $\geq 7$ & 3 & $1.19(1.12-1.28)$ & 0.447 & $0.0 \%$ & $<0.001$ \\
\hline Score $<7$ & 5 & $1.14(1.02-1.28)$ & 0.000 & $84.4 \%$ & 0.012 \\
\hline \multicolumn{6}{|l|}{ No of participants } \\
\hline$\geq 10000$ & 3 & $1.19(1.12-1.28)$ & 0.447 & $0.0 \%$ & $<0.001$ \\
\hline$<10000$ & 5 & $1.14(1.02-1.28)$ & 0.000 & $84.4 \%$ & 0.012 \\
\hline \multicolumn{6}{|l|}{ No of cases } \\
\hline$\geq 1500$ & 3 & $1.19(1.12-1.28)$ & 0.447 & $0.0 \%$ & $<0.001$ \\
\hline$<1500$ & 5 & $1.14(1.02-1.28)$ & 0.000 & $84.4 \%$ & 0.012 \\
\hline Colon cancer & 4 & $1.07(1.02-1.13)$ & 0.361 & $10.4 \%$ & $<0.001$ \\
\hline Renal cancer & 4 & $1.31(1.15-1.49)$ & 0.198 & $25.7 \%$ & $<0.001$ \\
\hline Malignant melanoma & 3 & $1.10(1.03-1.17)$ & 0.715 & $0.0 \%$ & $<0.001$ \\
\hline Brain cancer & 4 & $2.06(1.76-2.43)$ & 0.000 & $86.1 \%$ & $<0.001$ \\
\hline Esophagus cancer & 4 & $1.55(1.30-1.85)$ & 0.679 & $0.0 \%$ & $<0.001$ \\
\hline Prostate cancer & 3 & $1.26(1.16-1.37)$ & 0.000 & $79.5 \%$ & $<0.001$ \\
\hline Liver cancer & 3 & $1.22(1.13-1.31)$ & 0.000 & $62.9 \%$ & $<0.001$ \\
\hline Stomach cancer & 2 & $1.17(1.03-1.32)$ & 0.174 & $45.6 \%$ & $<0.001$ \\
\hline Pancreatic cancer & 2 & $1.39(1.17-1.64)$ & 0.813 & $0.0 \%$ & $<0.001$ \\
\hline Lung cancer & 3 & $1.20(1.12-1.28)$ & 0.000 & $89.8 \%$ & $<0.001$ \\
\hline
\end{tabular}


by inhibiting apoptosis and stimulating tumor cell proliferation [31]. Simultaneously, the treatment of benzodiazepine drug use before surgery can influence the depolarization of the mitochondrial membrane to inhibit the apoptosis of neutrophil cells, neutrophil apoptosis plays an important role in maintaining the homeostasis of the immune system and prevents damage to the host organs by promoting an immune response [32]. Addition, benzodiazepines are used to enhance the neurotransmitter of $\gamma$-aminobutyric acid by interacting with the chlorine ion channel that binds to GABA receptors. Besides as an inhibitory neurotransmitter effect, $\gamma$-aminobutyric acid is also thought can regulate cell proliferation and differentiation of brain and peripheral at various stages, and may participate in benign tumor growth in a variety of ways [33, 34]. However, the potential mechanisms of benzodiazepines and tumor growth still remain unclear and controversial.

To our knowledge, this is the first study to identify and quantify the potential dose-response association between benzodiazepine drug use and cancer risk in a large cohort of both men and women. Although, we performed this meta-analysis very carefully, however,
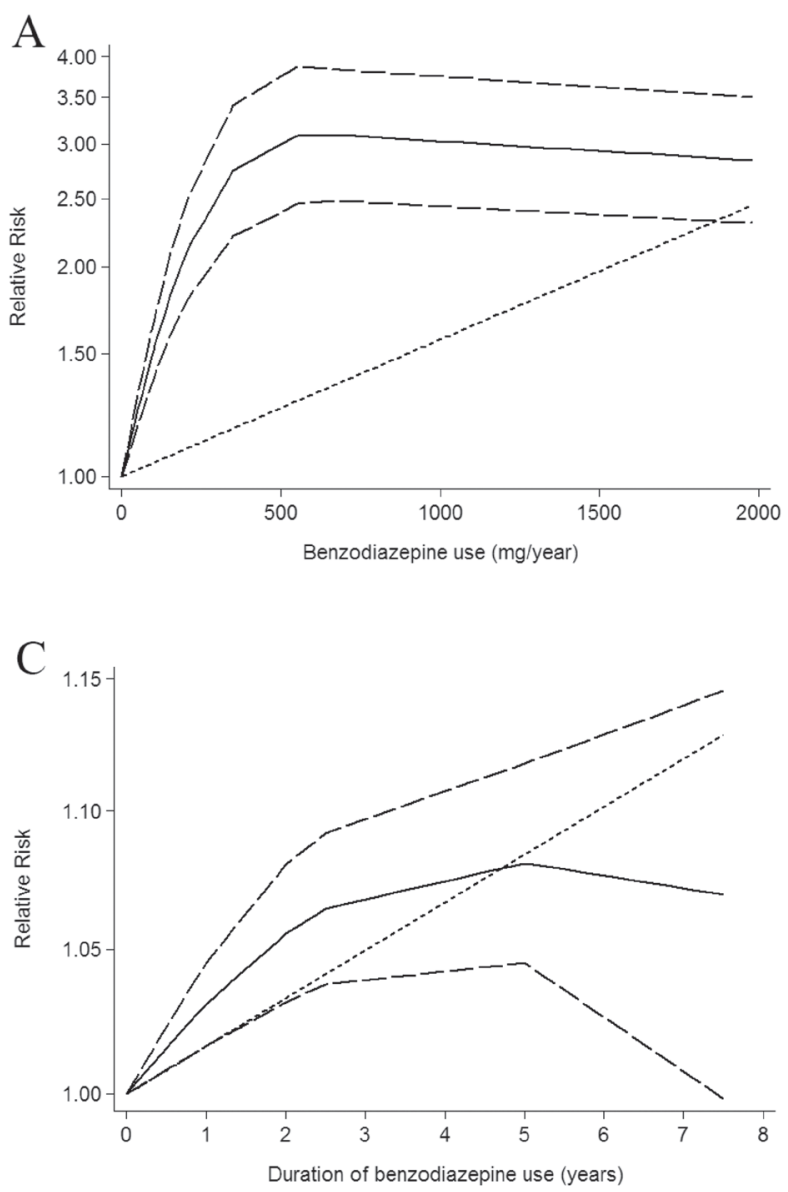

some limitations must be considered in the current metaanalysis. First, different sex of population should be included in this meta-analysis to explore the impact of different sex of population on benzodiazepine drug use and cancer risk. Second, we only select literature that written by English, which may have resulted in a language or cultural bias, other language should be chosen in the further. Third, in the subgroup analysis in cancer type, there has no insufficient statistical power to check a doseresponse in different cancer type, large data in different cancer type is warranted to validate this association.

In conclusion, our findings underscore the notion that benzodiazepine drug use was significantly associated with cancer risk increment. In the future, large-scale and population based association studies must be performed in the future to validate the risk identified in the current meta-analysis.

\section{MATERIALS AND METHODS}

This meta-analysis was conducted according to the Meta-analysis Of Observational Studies in Epidemiology (MOOSE) checklist [35].
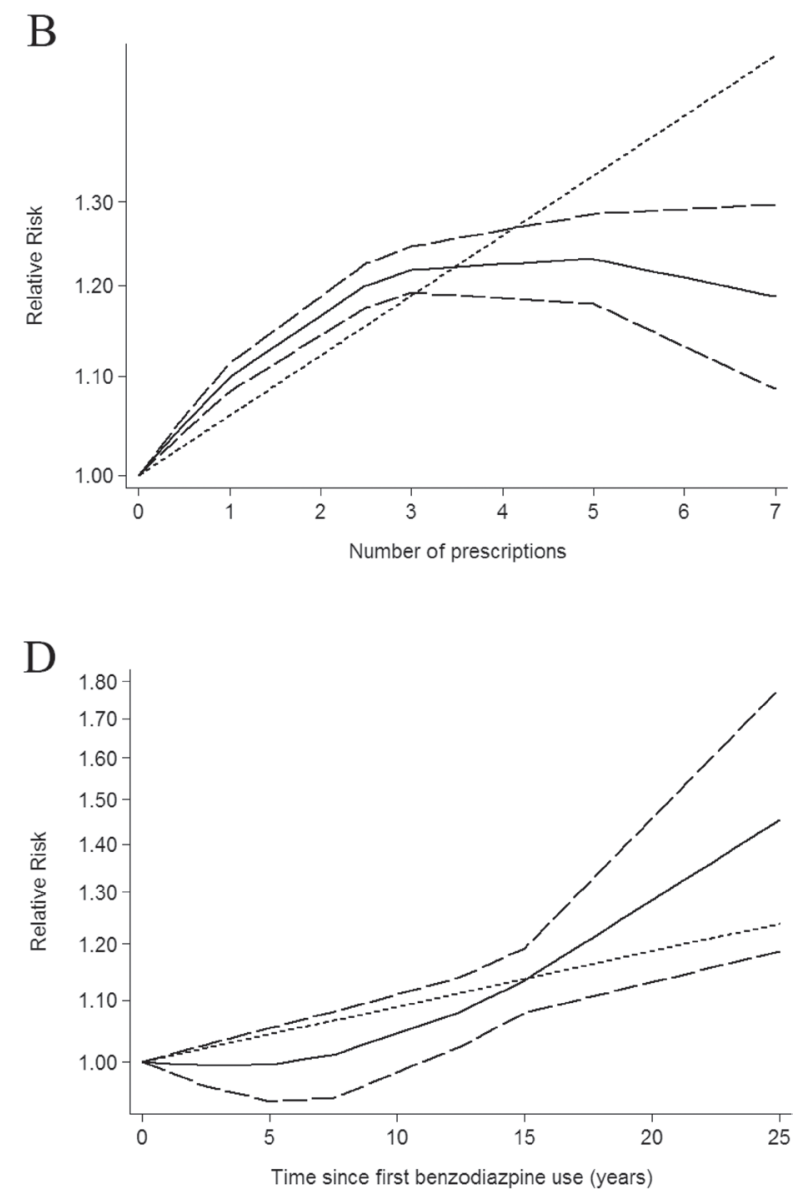

Figure 2: Dose-response relationship between benzodiazepine drug use in relation to risk of cancer. (A) Cumulative yearly dose of benzodiazepine drug use. (B) Number of prescriptions benzodiazepine drug use. (C) Duration of benzodiazepine drug use. (D) Time since first benzodiazepine drug use. 


\section{Search strategy}

We included eligible studies to investigate the relationship between benzodiazepines drug use and cancer risk. To develop a flexible, non-linear, $r$ meta-regression model, we required that an eligible study should have categorized into 3 or more levels.

PubMed, EMBASE and Web of Science databases were searched for studies that contained risk estimates for the outcomes of cancer and were published update to July 2017, with keywords including "benzodiazepine" [MeSH] OR "diazepam" [MeSH] OR "alprazolam" [MeSH] OR "clonazepam" $[\mathrm{MeSH}]$ OR "temazepam" $[\mathrm{MeSH}] \mathrm{OR}$ "oxazepam" [MeSH] AND “cancer" [MeSH] OR "tumor" [MeSH] "carcinoma" [MeSH] "neoplasm" [MeSH]. We refer to the relevant original essays and commentary articles to determine further relevant research.

\section{Study selection}

Two independent researchers investigate information the correlation between benzodiazepines drug use and cancer risk: outcome was cancer. Moreover, we precluded non-human studies, reviews, meta-analyses, editorials and published letters. To ensure the correct identification of qualified research, the two researchers read the reports independently.

\section{Data extraction}

Use standardized data collection tables to extract data. Each eligible article information was extracted by two independent researchers. We extracted the following information: first author; publication year; age; country; sex; cases and participants; the categories of benzodiazepines drug use; the relative risk or odds ratio (OR). We collect the risk estimates with multivariableadjusted. According to the Newcastle-Ottawa scale, quality assessment was performed for non-randomized studies [36].

\section{Statistical analysis}

We pooled relative risk estimates to measure the association between benzodiazepines drug use and cancer; the hazard ratio were considered equivalent to the relative risk [37]. Results in different subgroups of benzodiazepines drug use and cancer risk were treated as two separate reports.

Due to different definitions cut-off points in the included studies for categories, we performed a relative risk estimates by increaseper $500 \mathrm{mg} / \mathrm{year}$ of benzodiazepine using or per 3 prescriptions increment or per 3 year of duration of benzodiazepine using or per 5 year of time since first benzodiazepine use using the method recommended by Greenland, Longnecker and Orsini and colleagues. In addition, using restricted cubic splines to evaluate the non-linear association between benzodiazepines drug use and cancer risk, with three knots at the 10th, 50th, and 90th percentiles of the distribution. A flexible meta-regression based on restricted cubic spline (RCS) function was used to fit the potential non-linear trend, and generalized least-square method was used to estimate the parameters. This procedure treats benzodiazepines drug use (continuous data) as an independent variable and $\log R \mathrm{R}$ of diseases as a dependent variable, with both tails of the curve restricted to linear. A $P$ value is calculated for linear or non-linear by testing the null hypothesis that the coefficient of the second spline is equal to zero [38].

We use STATA software 12.0 (STATA Corp, College Station, TX, USA) to evaluate the relationships between benzodiazepines drug use and cancer risk. By using $\mathrm{Q}$ test and $\mathrm{I}^{2}$ statistic to assess heterogeneity among studies. Random-effect model was chosen if $P_{\mathrm{Q}}<0.10$ or $\mathrm{I}^{2}>50 \%$, otherwise, fixed-effect mode was applied. Sensitivity analysis was conducted to assess the stability of the results. Begg's and Egger's tests were to assess the publication bias of each study. $P<0.05$ was considered signifcant for all tests.

\section{CONFLICTS OF INTEREST}

Authors have disclosed no conflicts of interest.

\section{FUNDING}

This study received no specific external funding.

\section{REFERENCES}

1. GBD 2015 Mortality and Causes of Death Collaborators. Global, regional, and national life expectancy, all-cause mortality, and cause-specific mortality for 249 causes of death, 1980-2015: a systematic analysis for the Global Burden of Disease Study 2015. Lancet. 2016; 388:1459-544.

2. McGuire S. World Cancer Report 2014. Geneva, Switzerland: World Health Organization, International Agency for Research on Cancer, WHO Press, 2015. Adv Nutr. 2016; 7:418-9.

3. Kunert-Radek J, Stepien H, Pawlikowski M. Inhibition of rat pituitary tumor cell proliferation by benzodiazepines in vitro. Neuroendocrinology. 1994; 59:92-6.

4. Kim DH, Lee JT, Lee IK, Ha JH. Comparative anticancer effects of flavonoids and diazepam in cultured cancer cells. Biol Pharm Bull. 2008; 31:255-9.

5. Madhusoodanan S, Bogunovic OJ. Safety of benzodiazepines in the geriatric population. Expert Opin Drug Saf. 2004; 3:485-93.

6. Hogan DB, Maxwell CJ, Fung TS, Ebly EM. Prevalence and potential consequences of benzodiazepine use in senior citizens: results from the Canadian Study of Health and Aging. Can J Clin Pharmacol. 2003; 10:72-7. 
7. Coogan PF, Rosenberg L, Palmer JR, Strom BL, Stolley PD, Zauber AG, Shapiro S. Risk of ovarian cancer according to use of antidepressants, phenothiazines, and benzodiazepines (United States). Cancer Causes Control. 2000; 11:839-45.

8. Dublin S, Rossing MA, Heckbert SR, Goff BA, Weiss NS. Risk of epithelial ovarian cancer in relation to use of antidepressants, benzodiazepines, and other centrally acting medications. Cancer Causes Control. 2002; 13:35-45.

9. Fortuny J, Johnson CC, Bohlke K, Chow WH, Hart G, Kucera G, Mujumdar U, Ownby D, Wells K, Yood MU, Engel LS. Use of anti-inflammatory drugs and lower esophageal sphincter-relaxing drugs and risk of esophageal and gastric cancers. Clin Gastroenterol Hepatol. 2007; 5:1154-9 e3.

10. Friedman GD, Coates AO, Potter JD, Slattery ML. Drugs and colon cancer. Pharmacoepidemiol Drug Saf. 1998; 7:99-106. https://doi.org/10.1002/(sici)10991557(199803/04)7:2<99::aid-pds320>3.0.co;2-0.

11. Halapy E, Kreiger N, Cotterchio M, Sloan M. Benzodiazepines and risk for breast cancer. Ann Epidemiol. 2006; 16:632-6.

12. Hardell L, Fredrikson M, Axelson O. Case-control study on colon cancer regarding previous diseases and drug intake. Int J Oncol. 1996; 8:439-44.

13. Harlow BL, Cramer DW. Self-reported use of antidepressants or benzodiazepine tranquilizers and risk of epithelial ovarian cancer: evidence from two combined case-control studies (Massachusetts, United States). Cancer Causes Control. 1995; 6:130-4.

14. Harnod T, Lin CL, Sung FC, Kao CH. An association between benzodiazepine use and occurrence of benign brain tumors. J Neurol Sci. 2014; 336:8-12.

15. Jaussent I, Ancelin ML, Berr C, Peres K, Scali J, Besset A, Ritchie K, Dauvilliers Y. Hypnotics and mortality in an elderly general population: a 12-year prospective study. BMC Med. 2013; 11:212.

16. Kao CH, Sun LM, Su KP, Chang SN, Sung FC, Muo CH, Liang JA. Benzodiazepine use possibly increases cancer risk: a population-based retrospective cohort study in Taiwan. J Clin Psychiatry. 2012; 73:e555-60.

17. Kaufman DW, Shapiro S, Slone D, Rosenberg L, Helmrich SP, Miettinen OS, Stolley PD, Levy M, Schottenfeld D. Diazepam and the risk of breast cancer. Lancet. 1982; 1:537-9.

18. Kaufman DW, Werler MM, Palmer JR, Rosenberg L, Stolley PD, Warshauer ME, Clarke EA, Miller DR, Shapiro S. Diazepam use in relation to breast cancer: results from two case-control studies. Am J Epidemiol. 1990; 131:483-90.

19. Kleinerman RA, Brinton LA, Hoover R, Fraumeni JF Jr. Diazepam use and progression of breast cancer. Cancer Res. 1984; 44:1223-5.

20. Kripke DF, Langer RD, Kline LE. Hypnotics' association with mortality or cancer: a matched cohort study. BMJ Open. 2012; 2:e00850.
21. Lagergren J, Bergstrom R, Adami HO, Nyren O. Association between medications that relax the lower esophageal sphincter and risk for esophageal adenocarcinoma. Ann Intern Med. 2000; 133:165-75.

22. Landgren $\mathrm{O}$, Zhang $\mathrm{Y}$, Zahm SH, Inskip P, Zheng T, Baris D. Risk of multiple myeloma following medication use and medical conditions: a case-control study in Connecticut women. Cancer Epidemiol Biomarkers Prev. 2006; 15:2342-7.

23. Pogoda JM, Katz J, McKean-Cowdin R, Nichols PW, Ross RK, Preston-Martin S. Prescription drug use and risk of acute myeloid leukemia by French-American-British subtype: results from a Los Angeles County case-control study. Int J Cancer. 2005; 114:634-8.

24. Pottegard A, Friis S, Andersen M, Hallas J. Use of benzodiazepines or benzodiazepine related drugs and the risk of cancer: a population-based case-control study. Br J Clin Pharmacol. 2013; 75:1356-64.

25. Rosenberg L, Palmer JR, Zauber AG, Warshauer ME, Strom BL, Harlap S, Shapiro S. Relation of benzodiazepine use to the risk of selected cancers: breast, large bowel, malignant melanoma, lung, endometrium, ovary, non-Hodgkin's lymphoma, testis, Hodgkin's disease, thyroid, and liver. Am J Epidemiol. 1995; 141:1153-60.

26. Thygesen LC, Pottegard A, Ersboll AK, Friis S, Sturmer T, Hallas J. External adjustment of unmeasured confounders in a case-control study of benzodiazepine use and cancer risk. Br J Clin Pharmacol. 2017; 83:2517-2527.

27. Iqbal U, Nguyen PA, Syed-Abdul S, Yang HC, Huang CW, Jian WS, Hsu MH, Yen Y, Li YC. Is long-term use of benzodiazepine a risk for cancer? Medicine (Baltimore). 2015; 94:e483.

28. Westerdahl J, Olsson H, Masback A, Ingvar C, Jonsson $\mathrm{N}$. Risk of malignant melanoma in relation to drug intake, alcohol, smoking and hormonal factors. Br J Cancer. 1996; 73:1126-31.

29. Miyawaki I, Moriyasu M, Funabashi H, Yasuba M, Matsuoka N. Mechanism of clobazam-induced thyroidal oncogenesis in male rats. Toxicol Lett. 2003; 145:291-301.

30. Zdrinca M, Mutiu G, Bogdan M, Dobjanschi L, Antonescu A, Mos I, Muresan M, Zdrinca M, Antonescu A. Effects of Alprazolam, Zolpidem and Zopiclone, and of chronic inflammation on peripheral experimental algesia in Wistar rats. Rom J Morphol Embryol. 2011; 52:471-4.

31. Kaaks R, Lukanova A. Energy balance and cancer: the role of insulin and insulin-like growth factor-I. Proc Nutr Soc. 2001; 60:91-106.

32. Goto Y, O'Malley C, Fanning NF, Wang J, Redmond HP, Shorten GD. Benzodiazepines inhibit the rate of neutrophil apoptosis. Ir J Med Sci. 2003; 172:191-4.

33. Enna SJ. Role of gamma-aminobutyric acid in anxiety. Psychopathology. 1984; 17:15-24.

34. Jezewska E, Scinska A, Kukwa W, Sobolewska A, Turzynska D, Samochowiec J, Bienkowski P. Gammaaminobutyric acid concentrations in benign parotid tumours 
and unstimulated parotid saliva. J Laryngol Otol. 2011; 125:492-6.

35. Stroup DF, Berlin JA, Morton SC, Olkin I, Williamson GD, Rennie D, Moher D, Becker BJ, Sipe TA, Thacker SB. Meta-analysis of observational studies in epidemiology: a proposal for reporting. Meta-analysis Of Observational Studies in Epidemiology (MOOSE) group. JAMA. 2000; 283:2008-12.

36. Stang A. Critical evaluation of the Newcastle-Ottawa scale for the assessment of the quality of nonrandomized studies in meta-analyses. Eur J Epidemiol. 2010; 25:603-5.
37. Xu C, Zeng XT, Liu TZ, Zhang C, Yang ZH, Li S, Chen $\mathrm{XY}$. Fruits and vegetables intake and risk of bladder cancer: a PRISMA-compliant systematic review and dose-response meta-analysis of prospective cohort studies. Medicine (Baltimore). 2015; 94:e759.

38. Durrleman S, Simon R. Flexible regression models with cubic splines. Stat Med. 1989; 8:551-61. 